\title{
MODELAGEM ACÚSTICA BIDIMENSIONAL USANDO DIFERENTES PARAMETRIZAÇÕES DE CAMPOS DE VELOCIDADES
}

\author{
Roberto Hugo M. dos Santos $^{1}$ e Wilson M. Figueiró2 \\ Recebido em 19 dezembro, 2005 / Aceito em 14 março, 2006 \\ Received on December 19, 2005 / Accepted on March 14, 2006
}

\begin{abstract}
The algorithm developed, in this work, is based on the finite difference method applied to the acoustic wave equation, assuming that the Earth has an acoustic behavior, that allow us to implement, in a numerical way, a seismic modeling employing regular grids in models representing two-dimensional geological media. Second derivatives with respect to space and time of wave equation are obtained by Taylor series expansion of fourth and second orders, respectively. The models are represented by two different kinds of parameterizations: blocks and trigonometric polynomials. Simulations of the wave propagation phenomenon are accomplished in several models represented by the two mentioned parameterizations, making possible to generate synthetic seismograms to be compared. Seismograms, obtained when the polynomial parameterization is used, show some undesired behaviors such as: production of artificial reflections, weakening of reflection events, suppression of diffractions, and a little alteration in calculated traveltimes and amplitudes. By the other hand, some advantages of the proposed polynomial parameterization are: economy of computer memory space (because a complicated velocity model can be represented by a few quantity of coefficients, it means: the model is not more stored in a file, but it is compressed, or contained, in a mathematical formula or an analytical representation); production of a smooth velocity model (useful to generate a time field to be used in seismic migration, for example, in reverse time migration); the polynomial coefficients are just the parameters of the model to be estimated by an inversion procedure (independently of the degree of geometrical complication of the model and how is varying the seismic velocity on it); realistic models are better represented by trigonometric polynomial than block parameterization; and the ambiguity commonly present in inversion techniques results, is reduced if sufficient data is available. Gibbs effects, present in polynomial representations, is avoided by the finite differences method by choosing conveniently the knots of the mesh or increasing the number of polynomial coefficients.
\end{abstract}

Keywords: Velocity Field, Parameterization, Wave Equation, Finite-Difference Method, Seismograms.

RESUMO. 0 algoritmo desenvolvido neste trabalho é baseado no método das diferenças finitas aplicado à equação acústica da onda, assumindo que a Terra se comporta como um meio acústico, que permite implementar numericamente uma modelagem sísmica empregando malhas regulares em modelos que representam meios geológicos bidimensionais. As derivadas segundas presentes na equação da onda foram obtidas por expansões da série de Taylor de quarta ordem, para 0 caso espacial, e de segunda, para o temporal. Os modelos foram representados por dois tipos distintos de parametrizações: por blocos e polinomial trigonométrica. São apresentadas simulações do fenômeno de propagação de ondas em vários modelos, representados pelas duas parametrizações mencionadas, possibilitando, assim, a geração de sismogramas sintéticos para comparação. Os sismogramas obtidos, com a parametrização polinomial, mostram alguns comportamentos não desejados como: produção de reflexões artificiais, atenuação de reflexões, supressão de difrações, e pequenas alterações nos tempos de trânsito e amplitudes calculados. Por outro lado, como vantagens desta parametrização proposta, temos: economia de espaço de memória (pois um modelo complicado de velocidade pode ser representado por uma pequena quantidade de coeficientes, isto é: o modelo não é armazenado em um arquivo, mas é comprimido, ou contido, numa fórmula matemática ou numa representação analítica); produção de modelos de velocidades suavizadas (úteis na migração sísmica); sintetização do modelo num pequeno conjunto de coeficientes que se tornam parâmetros do modelo a serem estimados em possíveis procedimentos de inversão (independentemente do grau de complicação da geometria do modelo e da variação da velocidade sísmica); melhor representação de modelos realísticos por polinômios trigonométricos em comparação com a parametrização por blocos; e redução das ambigüidades normalmente presente nas técnicas de inversão, se uma quantidade suficiente de dados for disponível. Cabe acrescentar que: 0 efeito de Gibbs, presente em representações polinomiais, é contornado, no método das diferenças finitas, através da escolha conveniente dos nós da malha ou pelo aumento do número de coeficientes do polinômio.

Palavras-chave: Campo de Velocidade, Parametrização, Equação da Onda, Método das Diferenças Finitas, Sismogramas.

1,2CPGG-UFBA, Centro de Pesquisa em Geofísica e Geologia da Universidade Federal da Bahia, LAGEP, Instituto de Geociências, Rua Barão de Jeremoabo, sn, 40170290 Federação, Salvador, BA, Brasil. Fones: (55)(71) 3203-8522 e 3203-8520; Fax: (55)(71) 3203-8501 - E-mails: rhms@cpgg.ufba.br e figueiro@cpgg.ufba.br 


\section{INTRODUÇÃ̃O}

Métodos de soluções numéricas para a equação da onda foram estudadas por Mitchell (1969) e Boor (1972). Alford et al. (1974) comparou os resultados dos trabalhos usando diferenças finitas (DF) com resultados analíticos em modelos com quinas. Estes métodos atingem um alto grau de aproximaç̧ão, desde que se tenha uma grade suficientemente fina.

Vista como solução do problema direto na metodologia sísmica, a modelagem sísmica numérica pode ser empregada em: formulação da inversão sísmica não-linear, que requer uma teoria para a simulação de ondas sísmicas; processos de migração; auxílio à interpretação sísmica de regiões com geologia complexa; e geração de dados para testes em algoritmos de processamento.

Tradicionalmente, utiliza-se simplificações da equação da onda, como aproximações assintóticas e linearizações. Por exemplo: a teoria do raio (Červený et al., 1977; Keller, 1962) tem como base a equação eiconal, a qual é uma aproximação de alta frequiência (ótica geométrica), sendo inadequada quando o meio é altamente heterogêneo, apresentando problemas em modelos contendo interfaces com curvaturas abruptas (Trorey, 1970; Hilterman, 1970), entretanto com base no teorema de Green, ela permite a geração de resultados mais abrangentes, sendo útil para a modelagem de uma reflexão especíitica (evento). Como outro exemplo, temos os métodos espectrais, que apresentam limitações para modelos com geometria complexa e apresentam fortes gradientes de propriedades físicas, como acontece em contatos de litologias distintas.

0 uso do método das diferenças finitas, MDF, (Alterman \& Karal, 1968; Ottaviane, 1971) permite o desenvolvimento de técnicas com base na equação da onda sem qualquer aproximação ou limitação na geometria dos modelos. Desta forma, o método leva em conta não apenas as ondas diretas e refletidas (primárias e múltiplas), como também, ondas: superficiais, refratadas, convertidas, difratadas, e ondas observadas nas zonas de sombra da teoria do raio. O MDF permite, ainda, a obtenção de fotografias ("snapshots") do campo de ondas em qualquer instante da propagação.

0 MDF usa uma discretização do meio e, consequentemente, discretiza o campo de onda (amplitudes) que atravessa tal meio, usando uma malha de pontos. Esse método aproxima as derivadas espaciais e temporais por operadores de DF obtidos por uma expressão de Taylor truncada, usando pontos vizinhos em diferenças ponderadas. Seus resultados, tão precisos quanto desejado, apresentam acurácia muito superior em comparação aos outros métodos de modelagem sísmica.

\section{EQUAÇÃO ACÚSTICA DA ONDA}

Para os objetivos deste trabalho, é suficiente considerar meios acústicos na realização do estudo da influência que diferentes tipos de parametrizações do meio exercem na modelagem. Características distintivas básicas devem ser investigadas, preliminarmente em modelos representativos de meios menos complicados, além disso, espera-se que tais características se tornem cada vez mais acentuadas com o aumento do grau de complicação do meio e, consequentemente, do modelo que o representa.

Uma equação que relaciona a pressão com sua velocidade de propagação em meios acústicos é a chamada equação da onda de pressão e é dada por:

$$
\nabla^{2} p-\frac{1}{c^{2}} \frac{\partial^{2} p}{\partial t^{2}}=\vec{\nabla} \ln (\rho) \vec{\nabla} p
$$

onde $p=p(\mathbf{x}, t)$ representa a pressão, $\mathbf{x}$ é 0 vetor posição, $t$ é 0 tempo, $c$ é a velocidade de propagação da onda de pressão em $(\mathbf{x}, t), \rho$ é a densidade em $(\mathbf{x}, t), \nabla^{2} 0$ laplaciano relativo às dimensões espaciais, $\vec{\nabla}$ o operador gradiente, e $\ln 0$ logaritmo neperiano. Se $\rho$ é constante, então a Equação 1 ganha a forma:

$$
\nabla^{2} p-\frac{1}{c^{2}} \frac{\partial^{2} p}{\partial t^{2}}=0
$$

Esta equação é chamada de equação da onda escalar acústica. Temos que a relação pressão-deslocamento é dada por:

$$
P(\mathbf{x}, t)=-k \nabla \cdot \mathbf{u}(\mathbf{x}, t),
$$

onde $\mathbf{x}=(x, z), k$ é o módulo de compressão volumétrica, e $\mathbf{u}(\mathbf{x}, t)=\left(u_{1}(\mathbf{x}, t), u_{2}(\mathbf{x}, t)\right)$ é o vetor deslocamento do ponto material situado em $\mathbf{x}$ no instante $t$. Substituindo-se (3) em (2), chega-se à seguinte equação:

$$
\begin{aligned}
\operatorname{div}\left(\frac{\partial^{2} u_{1}}{\partial x^{2}}+\frac{\partial^{2} u_{1}}{\partial z^{2}}-\frac{1}{c^{2}} \frac{\partial^{2} u_{1}}{\partial t^{2}},\right. \\
\left.\frac{\partial^{2} u_{2}}{\partial x^{2}}+\frac{\partial^{2} u_{2}}{\partial z^{2}}-\frac{1}{c^{2}} \frac{\partial^{2} u_{2}}{\partial t^{2}}\right)=0 .
\end{aligned}
$$

sendo assim,

$$
\frac{\partial^{2} u_{1}}{\partial x^{2}}+\frac{\partial^{2} u_{1}}{\partial z^{2}}-\frac{1}{c^{2}} \frac{\partial^{2} u_{1}}{\partial t^{2}}=f_{1}(x, z, t)
$$

$$
\frac{\partial^{2} u_{2}}{\partial x^{2}}+\frac{\partial^{2} u_{2}}{\partial z^{2}}-\frac{1}{c^{2}} \frac{\partial^{2} u_{2}}{\partial t^{2}}=f_{2}(x, z, t)
$$

para $f_{1}$ e $f_{2}$ tais que:

$$
\frac{\partial f_{1}}{\partial x}=-\frac{\partial f_{2}}{\partial z} .
$$


Portanto, vamos considerar a equação escalar da onda, no caso bidimensional acústico, como:

$$
\frac{\partial^{2} u}{\partial x^{2}}+\frac{\partial^{2} u}{\partial z^{2}}=\frac{1}{c^{2}} \frac{\partial^{2} u}{\partial t^{2}}+f(x, z, t),
$$

onde $u=u_{2}(x, z, t)$ representa 0 deslocamento vertical do ponto material do meio relativamente à sua posição de equilíbrio $\mathbf{x}=(x, z)$ no instante $t, c=c(x, z)$ a velocidade de propagação da onda em $(x, z)$, e $f(x, z, t)$ a função fonte dada por:

$$
f(x, z, t)=0 \text { se }(x, z) \neq\left(x_{s}, 0\right) \text { e } f\left(x_{s}, 0, t\right)=f(t)
$$

com $x_{s}$ sendo a coordenada $x$ da posição da fonte.

\section{MÉTODO DAS DIFERENÇAS FINITAS}

Para substituir uma equação diferencial por uma equação que envolva somente diferenças e quocientes finitos (que não envolvem valores infinitamente grandes ou pequenos), que é o cerne do método, é necessário obter expressões que permitam calcular numericamente as derivadas. Isto pode ser feito via série de Taylor, que nos permite escrever, para a função $u=u(x)$, as seguintes expansões:

$$
\begin{aligned}
& u(x+\Delta x)=u(x)+\Delta x \cdot u^{(1)}(x)+ \\
& \quad+\frac{(\Delta x)^{2}}{2 !} u^{(2)}(x)+\frac{(\Delta x)^{3}}{3 !} u^{(3)}(x)+\ldots,
\end{aligned}
$$

e

$$
\begin{aligned}
& u(x-\Delta x)=u(x)-\Delta x \cdot u^{(1)}(x)+ \\
& \quad+\frac{(\Delta x)^{2}}{2 !} u^{(2)}(x)-\frac{(\Delta x)^{3}}{3 !} u^{(3)}(x)+\ldots,
\end{aligned}
$$

onde $u^{(n)}(x)$ representa a n-ésima derivada de $u$ relativamente a $x$.

Rearranjando os termos das Equações (9) e (10), obtemos então as seguintes séries para $u^{(1)}(x)$ :

$$
\begin{aligned}
u^{(1)}(x)= & \frac{[u(x+\Delta x)-u(x)]}{\Delta x}-\frac{\Delta x}{2 !} u^{(2)}(x) \\
& -\frac{(\Delta x)^{2}}{3 !} u^{(3)}(x)+\ldots,
\end{aligned}
$$

e

$$
\begin{aligned}
u^{(1)}(x)= & \frac{[u(x)-u(x-\Delta x)]}{\Delta x}+\frac{\Delta x}{2 !} u^{(2)}(x) \\
& -\frac{(\Delta x)^{2}}{3 !} u^{(3)}(x)+\ldots
\end{aligned}
$$

Truncando-se as Equações (11) e (12) a partir dos termos nos quais $\Delta x$ tem grau maior ou igual a 1 , obtemos as seguintes aproximações para a derivada primeira:

$$
u^{(1)}(x) \approx \frac{u_{i+1}-u_{i}}{h}
$$

e

$$
u^{(1)}(x) \approx \frac{u_{i}-u_{i-1}}{h}
$$

com $u_{i+1}=u(x+\Delta x), u_{i}=u(x), u_{i-1}=u(x-\Delta x)$ e $h=\Delta x$.

Podemos obter uma aproximação com menor erro de truncamento subtraindo-se a Equação (10) da Equação (9) e explicitando $u^{(1)}(x)$ :

$$
\begin{aligned}
u^{(1)}(x)= & \frac{1}{2}[u(x+h)-u(x-h)] h^{-1} \\
& -\frac{h^{2}}{3 !} u^{(3)}(x)-\frac{h^{4}}{5 !} u^{(5)}(x) .
\end{aligned}
$$

Truncando a série acima a partir do termo com $h^{2}$, obtemos a seguinte aproximação para $u^{(1)}(x)$ :

$$
u^{(1)}(x) \approx \frac{u_{i+1}-u_{i-1}}{2 h} .
$$

Podemos obter a derivada segunda subtraindo-se a Equação (12) da Equação (11) e explicitando $u^{(2)}(x)$, assim:

$$
u^{(2)}(x)=\frac{u_{i+1}-2 u_{i}+u_{i-1}}{h^{2}} .
$$

Usando as Equações (9) e (10) obtemos a seguinte expressão para a derivada segunda:

$$
\begin{aligned}
\left(u_{x x}\right)_{i}=\frac{1}{12 h^{2}}\left[-u_{i-2}\right. & +16 u_{i-1}-30 u_{i} \\
+ & \left.16 u_{i+1}-u_{i+2}\right],
\end{aligned}
$$

onde $u_{i+2}=u(x+2 \Delta x), u_{i-2}=u(x-2 \Delta x)$ e $\left(u_{x x}\right)_{i}$ é a derivada parcial segunda da função $u$ com respeito a $x$, calculada no nó $i$ da malha.

Usando a Equação (17), obtemos a seguinte aproximação para as derivadas segundas temporais:

$$
\left(u_{t t}\right)_{k}=\frac{1}{(\Delta t)^{2}}\left[u_{k-1}-2 u_{k}+u_{k+1}\right]
$$

onde $\left(u_{t t}\right)_{k}$ é a derivada segunda da função $u$ relativamente à variável $t$, calculada em $t=k \Delta t$, onde $\Delta t$ é 0 intervalo temporal.

Os operadores usados em DF executam uma ação específica sobre a função nos pontos da malha.

Em geral, quanto mais precisas as aproximações para as derivadas, mais pontos vizinhos são requeridos e, portanto, mais 
dispendioso torna-se o cálculo. No entanto, não só a precisão tem de ser levada em consideração, devemos, também, considerar critérios de estabilidade que são importantes para garantir convergência, que é assegurada por determinada largura da maIha espacial, assim como pelo intervalo temporal a ser usado.

As expressões (18) e (19) são, respectivamente, chamadas de aproximações de quarta ordem espacial e segunda ordem temporal da derivada segunda. A ordem da aproximação depende do número de pontos nas quais a função é amostrada. Tais pontos estão regularmente e simetricamente espaçados em relação àquele no qual se deseja conhecer a derivada segunda da função considerada.

\section{PROCEDIMENTO RECURSIVO DO MDF}

Utilizando-se as aproximações para as derivadas segundas representadas pelas Equações (18) e (19), obtemos a seguinte expressão recursiva necessária à implementação computacional do MDF aplicado à equação da onda:

$$
\begin{aligned}
& u_{i, j}^{k+1}=\frac{1}{12}\left(\frac{c \cdot \Delta t}{h}\right)^{2}\left[-\left(u_{i-2, j}^{k}+u_{i, j-2}^{k}\right)+\right. \\
& \quad+16\left(u_{i-1, j}^{k}+u_{i, j-1}^{k}\right)-60 u_{i, j}^{k}+ \\
& \left.\quad+16\left(u_{i+1, j}^{k}+u_{i, j+1}^{k}\right)-\left(u_{i+2, j}^{k}+u_{i, j+2}^{k}\right)\right] \\
& \quad+2 u_{i, j}^{k}-u_{i, j}^{k-1}+g,
\end{aligned}
$$

onde os índices $i, j$, e $k$ representam, respectivamente, as variáveis $x$, $z$ e $t$; e $g=-(c \cdot \Delta t)^{2} f$.

\section{CONDIÇÃO PARA ESTABILIDADE E NÃO DISPERSÃO NUMÉRICA}

No MDF, o cálculo das derivadas envolvidas nas equações diferenciais são realizados segundo aproximações com maior ou menor precisão, o que gera erro no resultado numérico. No caso particular da equação da onda, este erro se apresenta sobre a forma de dispersão numérica. Este problema geralmente se manifesta gerando oscilações intermitentes na forma temporal do pulso sísmico. Outro problema causado é a presença de ruído em um determinado ponto antes da chegada do sinal.

Para que haja estabilidade, ou seja, para que o erro na determinação das amplitudes das ondas permaneça pequeno e não cresça com o tempo, faz-se necessário calcular o intervalo de amostragem temporal $(\Delta t)$ máximo que seja mais conveniente.

Segundo Cunha \& Mufti (1997), a modelagem por DF emprega freqüentemente malha uniforme $(\Delta x=\Delta z)$, que, vi- sando evitar dispersão ou instabilidade numéricas, deve atender às relações que seguem:

$$
\Delta x=\Delta z \leq \frac{c_{\min }}{\alpha \cdot f_{\max }}
$$

$$
\Delta t=\frac{\Delta x}{\beta \cdot c_{\max }},
$$

onde $\Delta x$ e $\Delta z$ são os intervalos espaciais; $\Delta t$ é 0 intervalo temporal utilizado; $c_{\min }, c_{\max }$ são, respectivamente, as velocidades mínima e máxima presentes no modelo; $f_{\max }$ é a freqüência máxima da fonte; e as constantes $\alpha$ e $\beta$ são parâmetros que dependem da ordem de aproximação usada para cálculo das derivadas espaciais e temporais. A qualidade do resultado melhora com 0 aumento de $\alpha$.

\section{FUNÇÃO FONTE}

Um conceito muito discutido quando se fala em resolução vertical é o poder de resolução, $P_{a}$, do pulso sísmico. Fisicamente é uma medida do quanto um pulso de banda limitada tende a se aproximar de uma função impulso perfeita. Para estudos de resolução é mais conveniente que seja usado um pulso de fase zero. Este pode ser dado diretamente no domínio do tempo ou pode ser definido a partir de seu espectro de freqüências. Porém, é conveniente que seja uma função bem comportada, com espectro limitado ou que decaia rapidamente a partir de um determinado ponto, de modo que uma freqüência dominante, assim como a de corte, fiquem bem caracterizadas. É bom enfatizar ser necessário estrito controle sobre o conteúdo de freqüência da fonte quando se trabalha com DF. Desta forma, se pode saber qual a largura da malha que mantém a dispersão sob controle. Neste trabalho foi utilizada como função fonte o pulso que possui a forma da segunda derivada da função gaussiana, definida pela fórmula:

$$
f(t)=\left[1-2\left(\pi f_{p} t\right)^{2}\right] e^{-\left(\pi f_{p} t\right)^{2}}
$$

onde $f_{p}$ é a freqüência de pico. A Figura 1 ilustra a função $f(t)$, que foi usada como fonte.

Nos experimentos realizados neste trabalho a fonte foi colocada na posição $550 m$ na superfície de observação com uma freqüência máxima de $80 \mathrm{~Hz}$. 


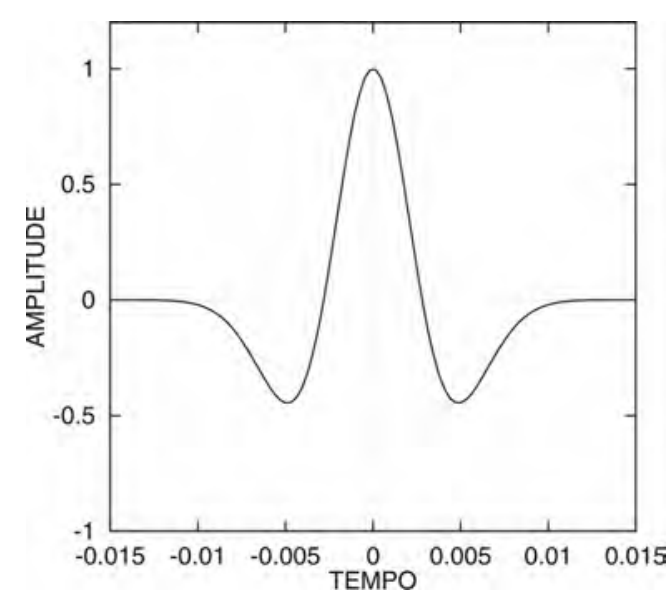

Figura 1 - Pulso com forma da derivada segunda da função gaussiana usado como função fonte.

\section{REFLEXÃO NAS BORDAS}

Qualquer aproximação por DF, bem como qualquer outro método de solução numérica da equação da onda, enfrenta problemas com as bordas do modelo. Uma solução, no caso de fonte pontual, é a aproximação explícita, que consiste em estender os limites do modelo, de modo que estas reflexões indesejadas não tenham tempo de estar presente no sismograma. Porém, este método é muito caro computacionalmente. Outra maneira de atacar este problema é tentar anular as reflexões impondo-se condições de contorno apropriadas nas referidas bordas. 0 esquema aqui utilizado, Cerjan et al. (1985), aplica, a cada iteração, uma diminuição gradativa das amplitudes em uma faixa nas proximidades de cada borda. Pontos a uma distância $i$ da borda tem sua amplitude $P_{0}$ reduzida para $P$ através de:

$$
(P)_{i}=\left(P_{0}\right)_{i} \cdot \exp \left[-F(N-i)^{2}\right]
$$

onde $F$ é o fator de atenuação; $N$ é o número de pontos da malha entre a fonte e a borda do modelo; e $i$ é a distância, em pontos da malha, até a borda do modelo. Cerjan et al. (1985) sugere $N=20$ e $F=0,015$.

\section{DEFINIÇÃO DE MODELOS SÍSMICOS 2-D}

Os modelos considerados têm dimensão horizontal de $1100 \mathrm{~m}$ e profundidade de $600 \mathrm{~m}$ sendo os modelos sísmicos com graus de complexibilidade crescente na seguinte seqüência: $M_{I}$ (de duas camadas homogêneas separadas por uma interface horizontal, Figura 2), modelo $M_{I I}$ (de seis camadas homogêneas, Figura 3), $M_{I I I}$ (apresenta variações laterais e verticais, Figura 4), $M_{I V}$ (constituído por quatro camadas e dotado de uma intrusão, Figura 5), $M_{V}$ (de três camadas com uma falha normal, Figura 6), e $M_{V I}$ (de três camadas e uma bacia, Figura 7). Sismogramas são gerados para cada modelo, que são representados por duas diferentes parametrizações: por blocos (PB) e pela polinomial trigonométrica (PPT)

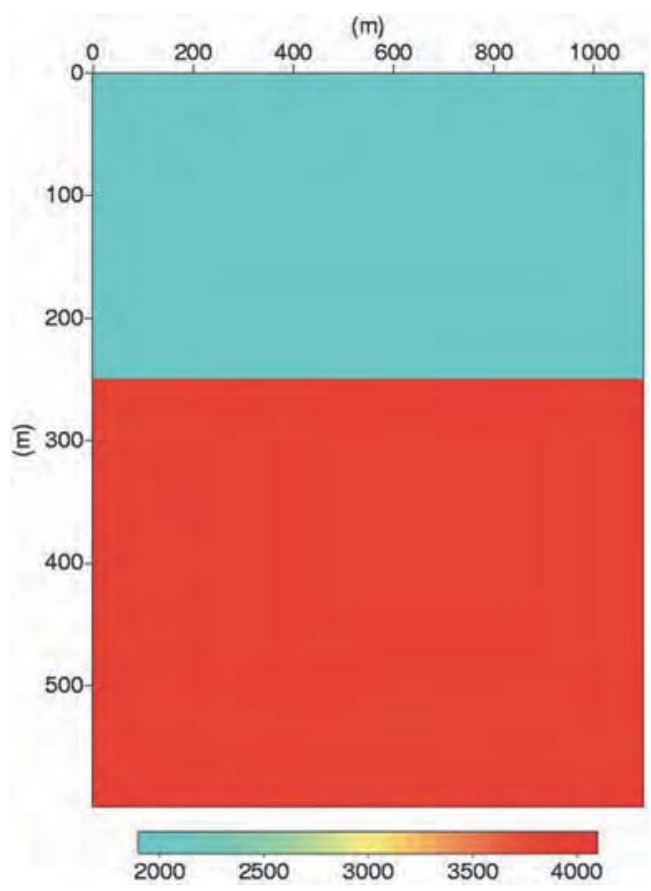

Figura 2 - Modelo de campo de velocidades sísmicas compressionais $\left(M_{I}\right)$ com duas camadas homogêneas isotrópicas separada por uma interface horizontal situada a, aproximadamente, $250 \mathrm{~m}$ de profundidade.

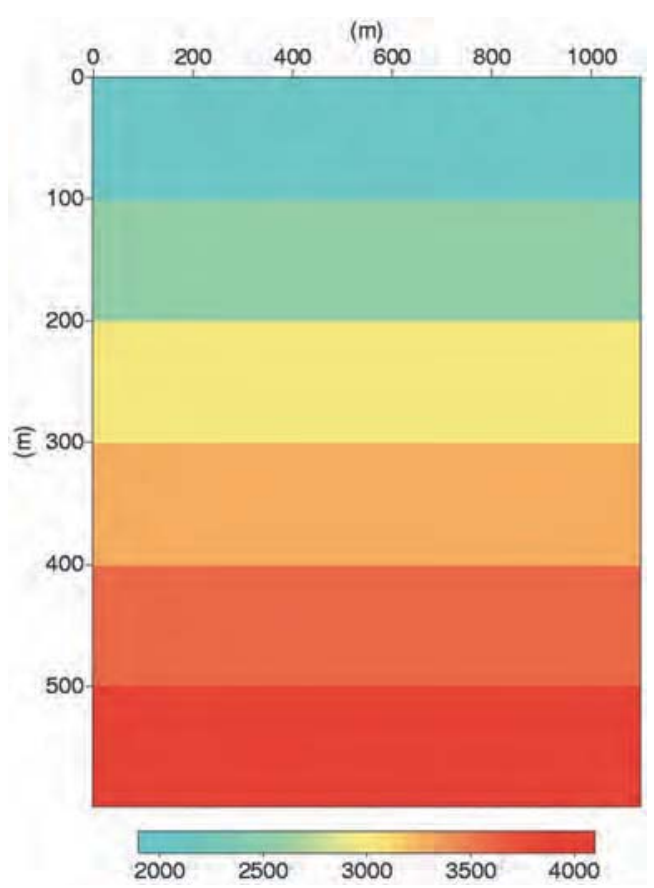

Figura 3 - Modelo de campo de velocidades sísmicas compressionais $\left(M_{I I}\right)$ constituído por seis camadas homogêneas isotrópicas com $100 \mathrm{~m}$ de espessura cada uma. 


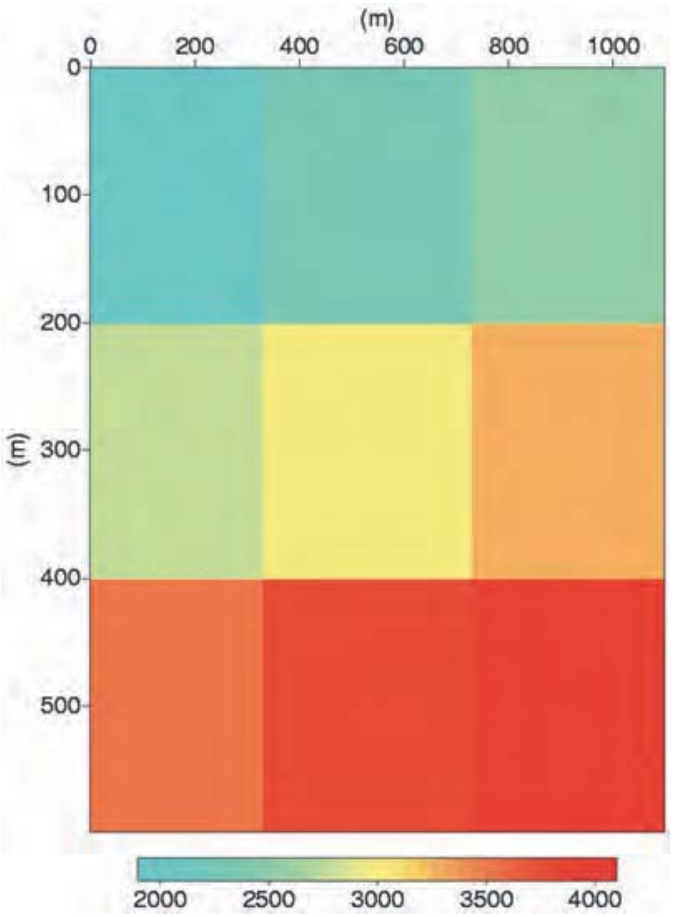

Figura 4 - Modelo de campo de velocidades sísmicas compressionais $\left(M_{I I I}\right)$ dotado de variações laterais e verticais. Tal modelo é constituído por nove regiões: retangulares, homogêneas, e isotrópicas; separadas por interfaces horizontais e verticais. Todas estas regiões têm as mesmas dimensões. As variações horizontais são mais suaves que as verticais.

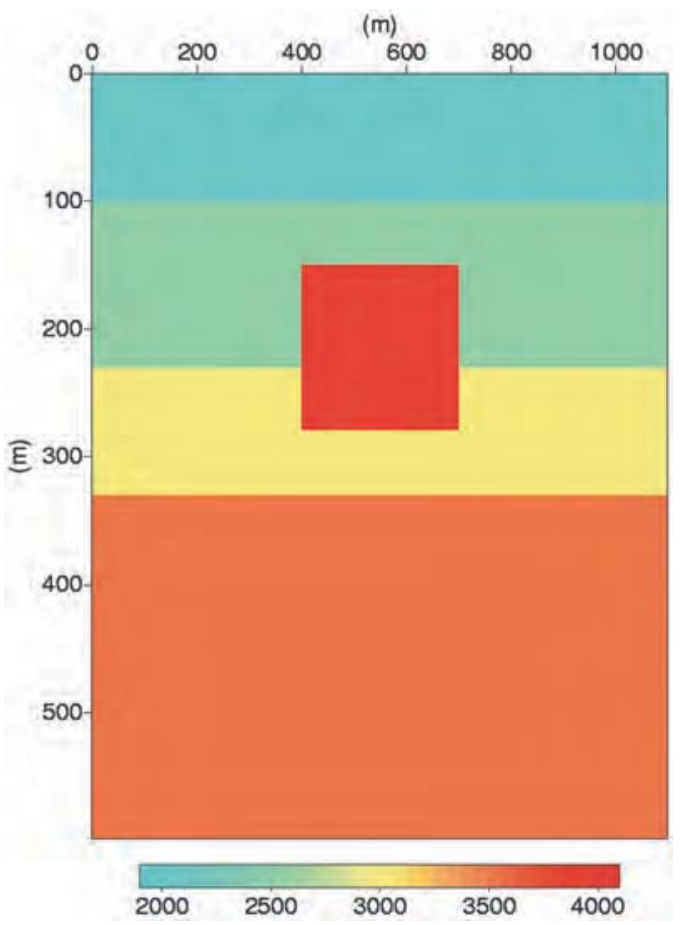

Figura 5 - Modelo de campo de velocidades sísmicas compressionais $\left(M_{I V}\right)$ constituído por quatro camadas e uma intrusão retangular de alta velocidade, todas homogêneas e isotrópicas. As interfaces entre as camadas são horizontais.

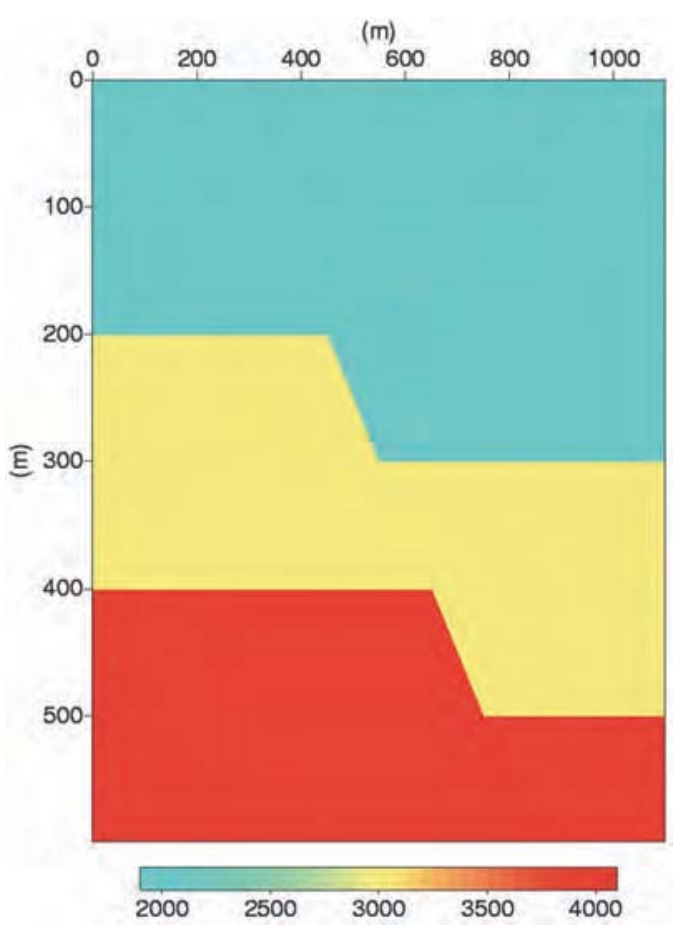

Figura 6 - Modelo de campo de velocidades sísmicas compressionais $\left(M_{V}\right)$ constituído por três camadas desalinhadas por uma falha geológica normal esquemática. As camadas são homogêneas e isotrópicas.

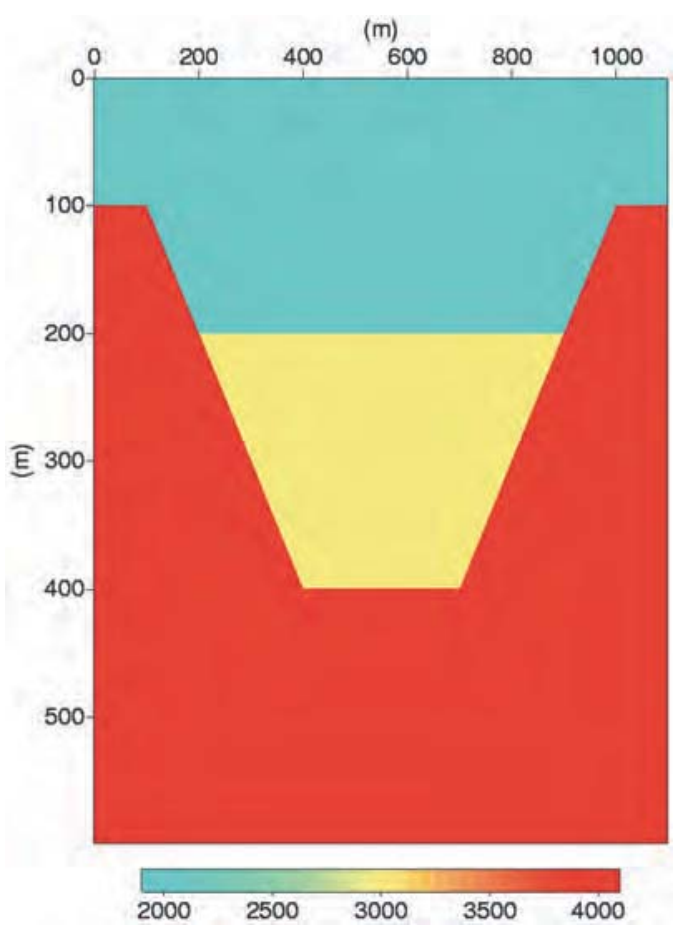

Figura 7 - Modelo de campo de velocidades sísmicas compressionais $\left(M_{V I}\right)$ representando uma visão esquemática de uma bacia geológica. As diferentes regiões do modelo são homogêneas e isotrópicas. 


\section{PARAMETRIZAÇÕES DE CAMPOS DE VELOCIDADES SÍSMICAS}

\section{Divisão do meio em Células}

A utilização de células (ou blocos) na representação do modelo é o tipo de parametrização mais utilizado na modelagem sísmica, que é, simplesmente, a divisão do modelo em blocos (ou células), onde cada um possui uma velocidade constante. As Figuras 8, e 9 exibem, respectivamente, os modelos $M_{I V}$, e $M_{V I}$; parametrizados por blocos. Nesta parametrização o número de blocos totalizam $19 \times 26=494$, onde cada um tem, aproximadamente, $58 m$ de comprimento e $23 m$ de largura. Cada bloco é homogêneo, isotrópico, e com velocidade igual àquela da região do modelo por ele coberta.

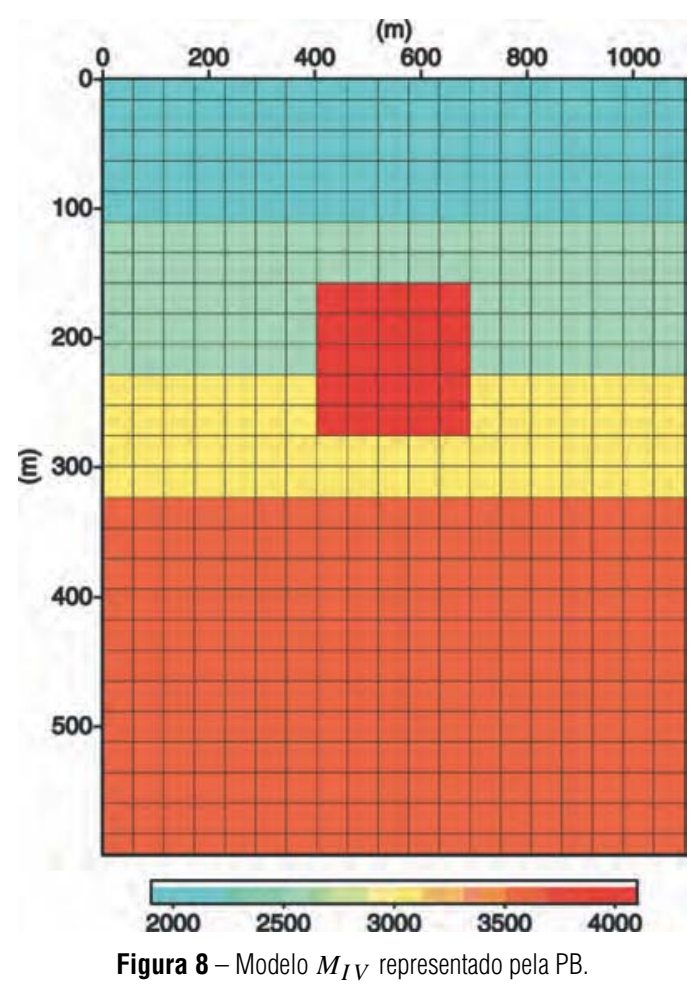

\section{Polinômio Trigonométrico}

A parametrização polinomial trigonométrica consiste em representar o modelo geofísico através de uma única função polinomial trigonométrica. Tal representação é obtida através da particularização do teorema que fornece séries baseadas em funções ortogonais para funções de duas variáveis (Kreider et al., 1972). Isto é, o campo de velocidade sísmica $V(x, z)$ é representado por:

$$
V(x, z)=\sum_{i+j=0}^{N} \alpha_{i, j} f_{i}(x) g_{j}(z),
$$

onde

$$
\begin{gathered}
f_{i}(x)=\frac{1}{2}\left\{\left[(-1)^{i}+1\right] \cos \left(\frac{i X}{2}\right)+\right. \\
\left.+\left[(-1)^{i+1}+1\right] \operatorname{sen}\left[\frac{(i+1) X}{2}\right]\right\}, \\
g_{j}(z)=\frac{1}{2}\left\{\left[(-1)^{j}+1\right] \cos \left(\frac{j Z}{2}\right)+\right. \\
\left.+\left[(-1)^{j+1}+1\right] \operatorname{sen}\left[\frac{(j+1) Z}{2}\right]\right\}, \\
X=\frac{\pi(2 x-l)}{l}, \\
\alpha_{i, j}=\frac{\pi(2 z-d)}{d}, \\
\iint_{R} V(x, z) f_{i}(x) g_{j}(z) d R
\end{gathered}
$$

onde $R$ é a região espacial na qual o modelo é considerado, e $l$ e $d$ representam, respectivamente, o comprimento e a profundidade máximas do modelo. Tal função representa o campo de velocidades do modelo no qual a onda se propaga.

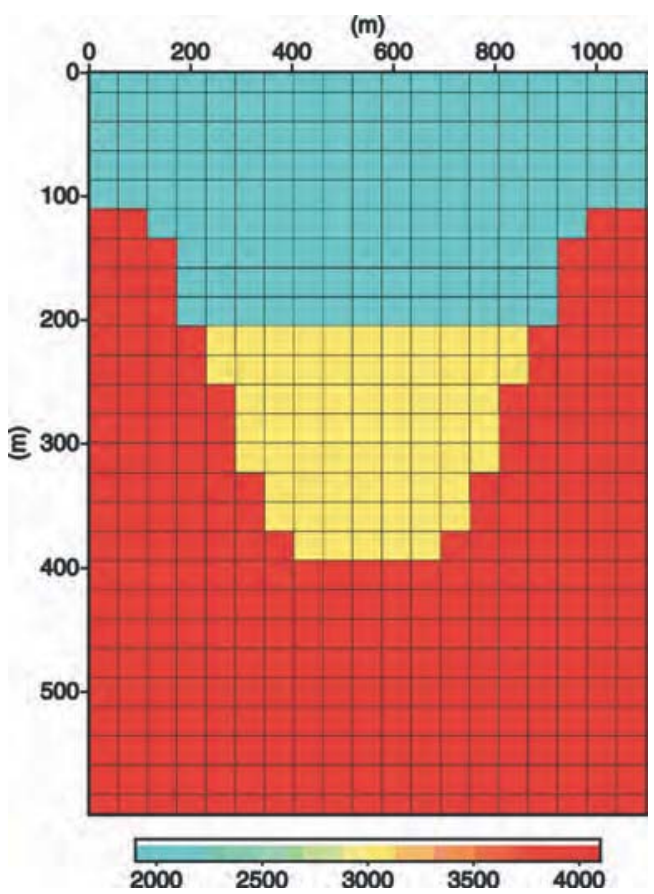

Figura 9 - Modelo $M_{V I}$ representado pela PB. As interfaces inclinadas adquiriram uma forma de escada.

Para todos os modelos sísmicos parametrizados pelo polinômio trigonométrico foram utilizados 80 coeficientes. Para modelos mais simples tal número pode parecer um exagero, 
entretanto, à medida que vamos tendo modelos mais complicados, ou seja, com um número cada vez maior de parâmetros, 0 número de coeficientes utilizados vai ajustar-se à necessidade de representação desses modelos. 0 número 80 foi mantido em todos os modelos visando uma interpretação uniformizada dos resultados.

Com 0 objetivo de facilitar os cálculos das integrais, foram feitas aproximações com pequenos quadrados nas proximidades das interfaces inclinadas tanto para o modelo $M_{V}$ como para 0 modelo $M_{V I}, 0$ que nos dá a certeza de que, independentemente da complexibilidade dos modelos, este tipo de parametrização pode ser utilizada.

As Figuras 10 e 11 mostram, respectivamente, os modelos $M_{I V}$, e $M_{V I}$; parametrizados por polinômios trigonométricos. Neles observamos, no interior das regiões homogêneas, oscilações características das funções trigonométricas. Além disso, nas interfaces podemos observar efeitos devido ao fenômeno de Gibbs.

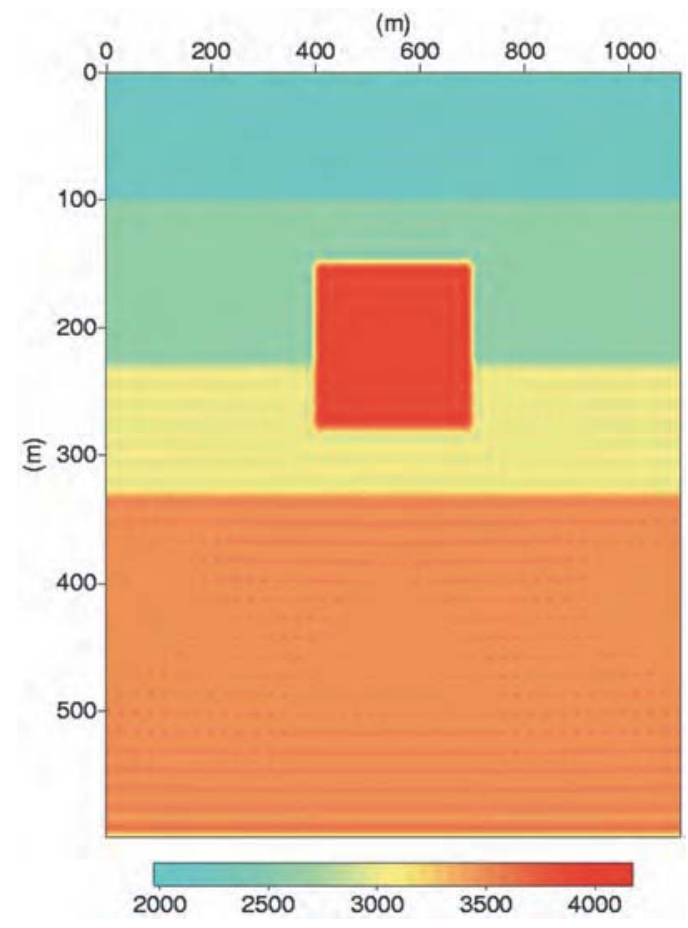

Figura 10 - Modelo $M_{I V}$ representado pela PPT.

Dentre as vantagens da parametrização polinomial temos: economia de espaço de memória; os polinômios podem ser, facilmente e infinitamente, derivados e integrados; todos os aspectos do modelo ficam resumidos nos coeficientes do polinômio; possíveis trabalhos de inversão ficam sintetizados na estimativa dos coeficientes do polinômio; e se o campo de velocidades for contínuo (ou possuir descontinuidades de salto finito), então existe uma seqüência de funções polinomiais trigonométricos que para ele converge.

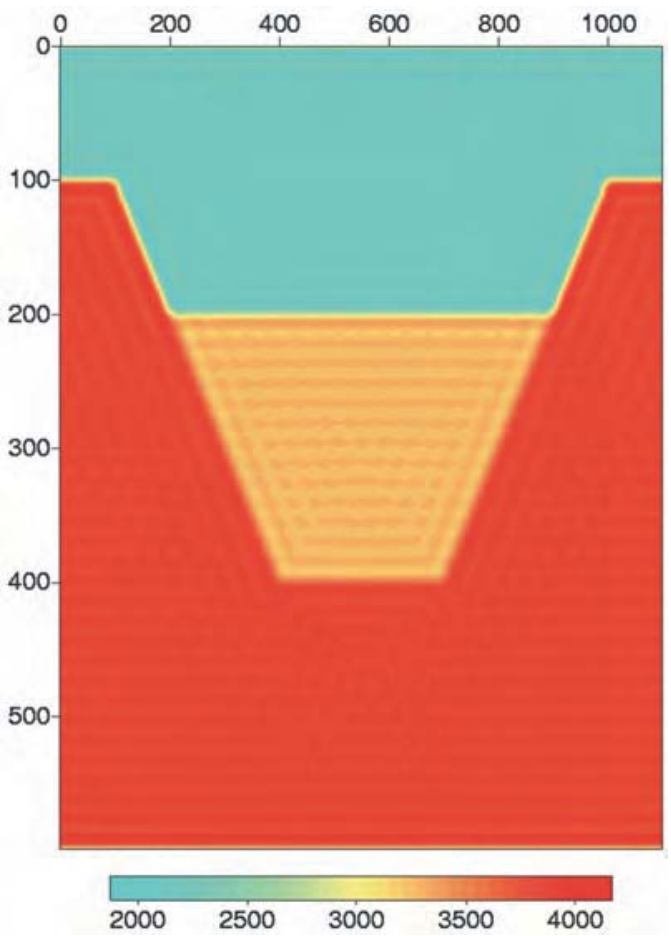

Figura 11 - Modelo $M_{V I}$ representado pela PPT.

\section{MODELAGEM ACÚSTICA 2-D}

Foram realizadas modelagens acústicas baseadas em operadores escalares gerando doze sismogramas para os seis modelos propostos utilizando-se as duas parametrizações consideradas para cada um deles. Foram implementados programas Fortran para simulação do campo de onda acústico. Tal simulação foi realizada adotando-se: o MDF com aproximação de segunda ordem no tempo e quarta ordem no espaço, malhas regulares, condições de contorno não reflexivas (versão "one-way" da equação da onda) conjugadas com bordas de absorção como propostas por Cerjan et al. (1985), e termo fonte proveniente da segunda derivada da função gaussiana, como definido em Cunha \& Mufti (1997). Além disso, para garantir estabilidade numérica e evitar dispersão de energia, os intervalos de amostragem foram definidos por 10 amostras por comprimento de onda (temporal e espacial).

Para os modelos considerados na seqüência de $M_{I}$ a $M_{V I}$, as Figuras 12, 13, 14, 15, 16, e 17 mostram em (a) os sismogramas sintéticos obtidos por modelagem acústica aplicando o MDF à equação da onda, usando blocos (PB), enquanto em (b), mostram aqueles produzidos quando os modelos são representados pela função polinomial trigonométrica. 


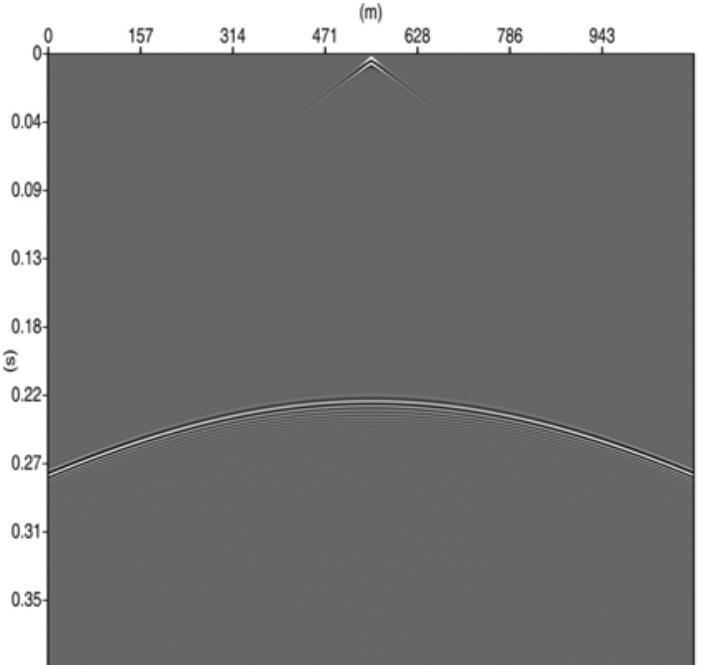

(a)

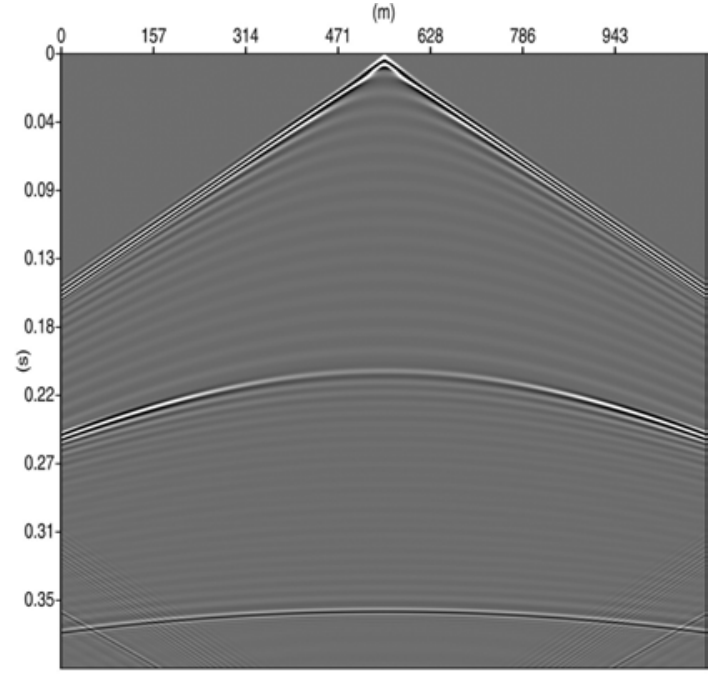

(b)

Figura 12 - Sismogramas sintéticos referentes ao modelo $M_{I}$, usando: (a) PB, e (b) PPT. A reflexão que aparece na parte inferior de (b) se deve às oscilações da PPT nas proximidades da borda inferior de $M_{I}$.

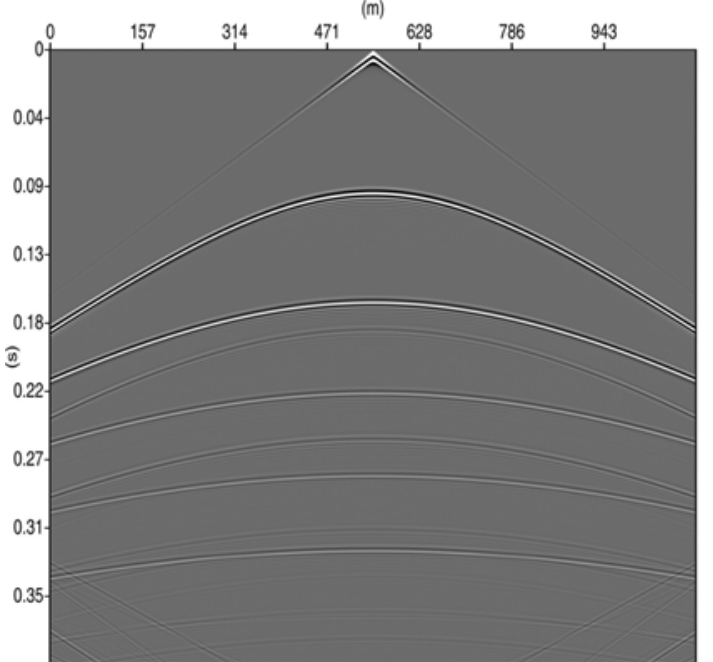

(a)

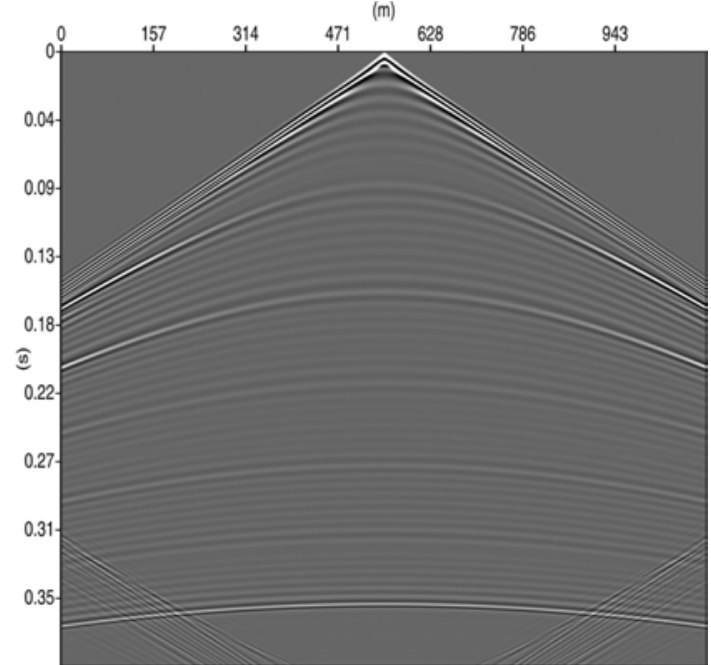

(b)

Figura 13 - Sismogramas sintéticos referentes ao modelo $M_{I I}$, usando: (a) PB, e (b) PPT. Em (a) as reflexões nas cinco interfaces podem ser claramente identificadas, enquanto que em (b) elas aparecem em meio a inúmeros eventos reflexivos artificiais.

\section{ANÁLISE DOS RESULTADOS}

Na Figura 12 os eventos se manifestam com maior clareza em (a) do que em (b). A primeira reflexão na interface de $M_{I}$ revela um pequeno deslocamento vertical quando comparamos (a) com (b). Pode-se observar também que as oscilações do polinômio trigonométrico cria fracas reflexões.

Na Figura 13, a chegada da onda direta apresenta-se com maior clareza em (b), entretanto as primeiras reflexões em cada interface manifestam-se de modo mais nítido em (a), além disso, no sismograma (a), obtido com a PB, são visíveis as múltiplas referentes às duas primeiras interfaces, enquanto que na PPT, sismograma (b), estas múltiplas não são facilmente visíveis.

$\mathrm{Na}$ Figura 14, em (a), além das ondas diretas, primeiras reflexões nas interfaces e a múltipla da primeira interface, também podem ser observadas as difrações nas quinas do modelo $M_{I I I}$. Entretanto, em (b), estas difrações são atenuadas devido à suavização das quinas, do mesmo modelo sísmico, causada pela PPT. 


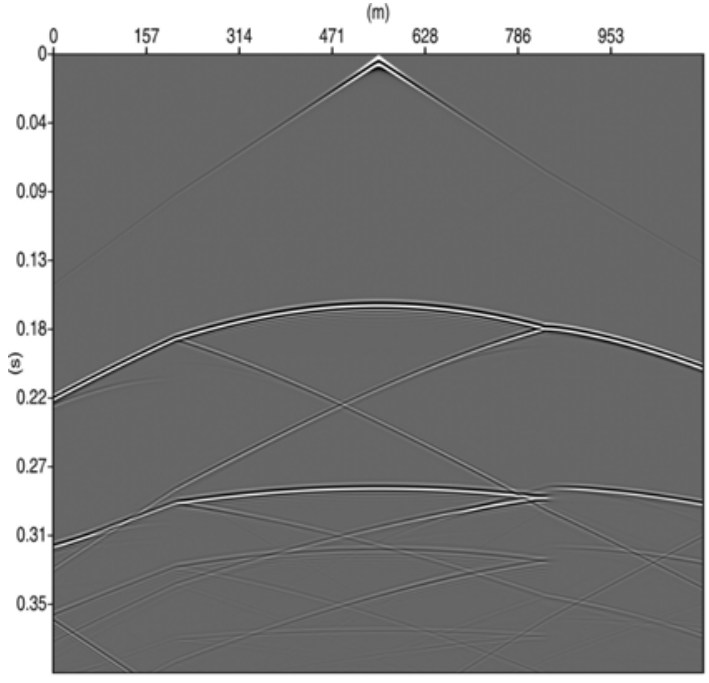

(a)

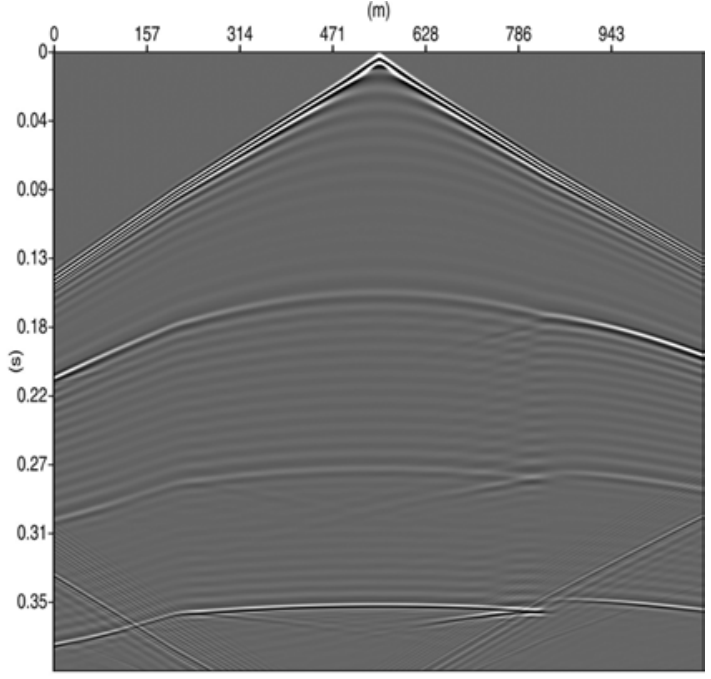

(b)

Figura 14 - Sismogramas sintéticos referentes ao modelo $M_{I I I}$, usando: (a) PB, e (b) PPT. Em (a), entre as reflexões na primeira e segunda interfaces, vemos eventos relativos às interfaces verticais.

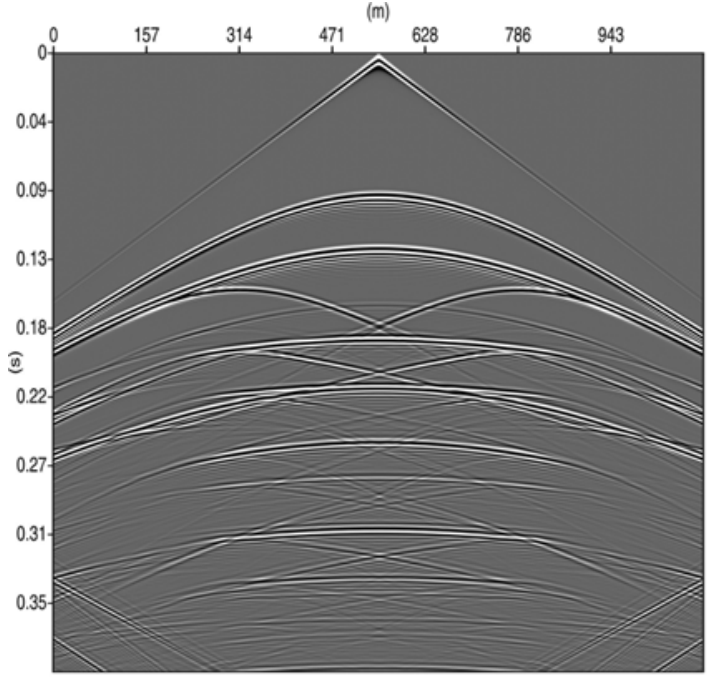

(a)

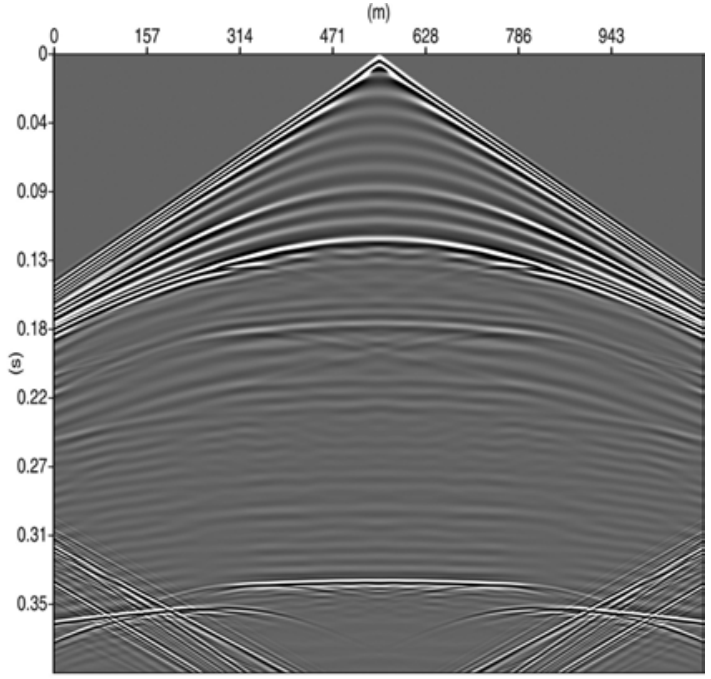

(b)

Figura 15 - Sismogramas sintéticos referentes ao modelo $M_{I V}$, usando: (a) PB, e (b) PPT. Em (a), o uso da PPT dificulta a identificação de vários eventos associados à intrusão.

Na Figura 15, todos os eventos em (a) são facilmente identificados enquanto em (b) o refletor acima da base e do topo da intrusão não são identificados.

Observa-se em (a) da Figura 16, que as reflexões nas interfaces são prolongadas pelas difrações nas quinas do modelo $M_{V}$ e pelas reflexões no plano de falha, ocorrendo dessa forma 0 cruzamento de eventos. Já em (b), onde as quinas difratoras são suavizadas, não ocorre este prolongamento e sim uma descontinuidade causada pelo plano de falha.
Na Figura 17, os resultados, de certa forma, são semelhantes àqueles mostrados na Figura 16, pois de forma análoga 0 sismograma (a) apresenta eventos contínuos causados pelas difrações nas quinas do modelo $M_{V I}$ e reflexões nas paredes da bacia, enquanto que em (b) as difrações são atenuadas e as reflexões do fundo da bacia só são registradas nos geofones centrais.

É importante citar que nas comparações entre os sismogramas dos diferentes modelos, existe uma diferença no posicionamento dos refletores causada pelas diferentes velocida- 


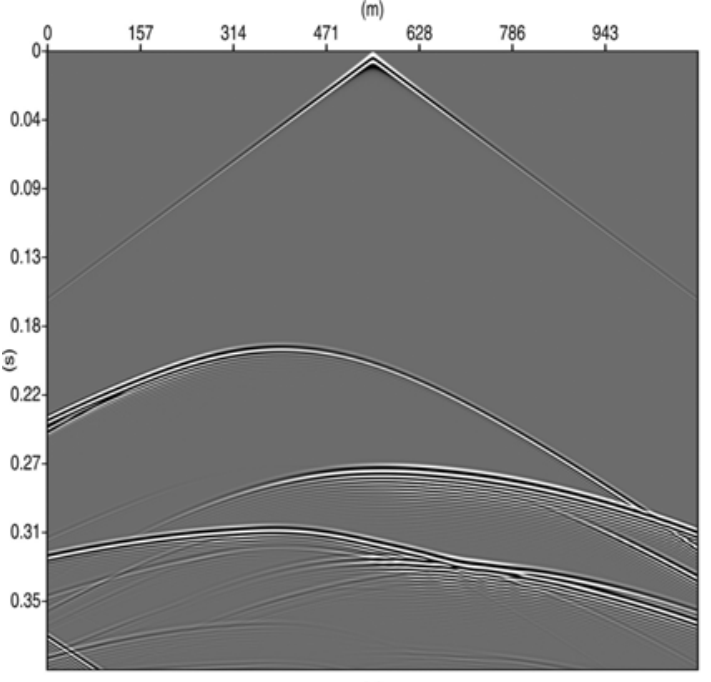

(a)

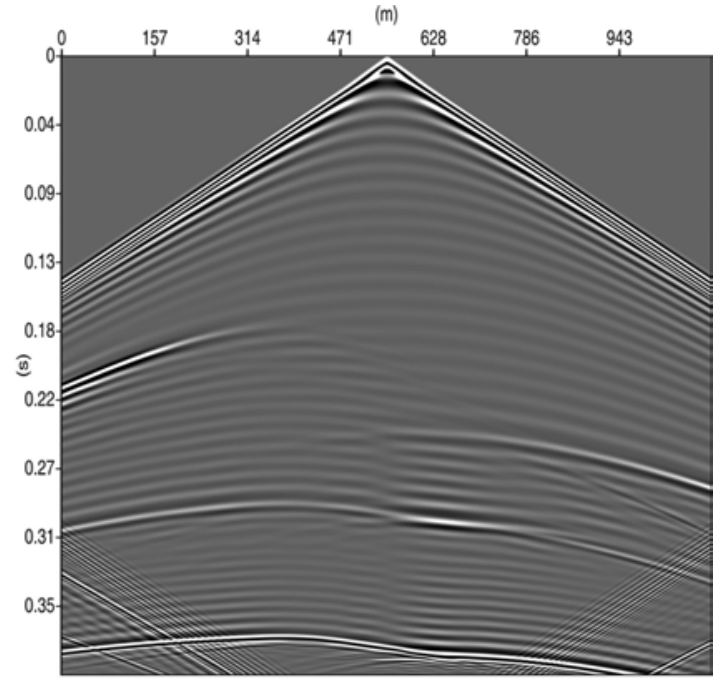

(b)

Figura 16 - Sismogramas sintéticos referentes ao modelo $M_{V}$, usando: (a) PB, e (b) PPT. Em (a), a assimetria do modelo se manifesta nos sismogramas.

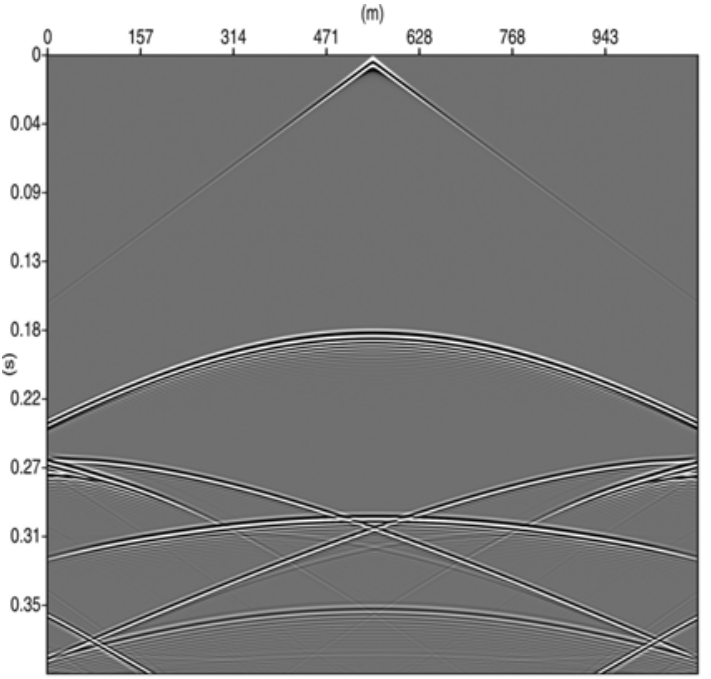

(a)

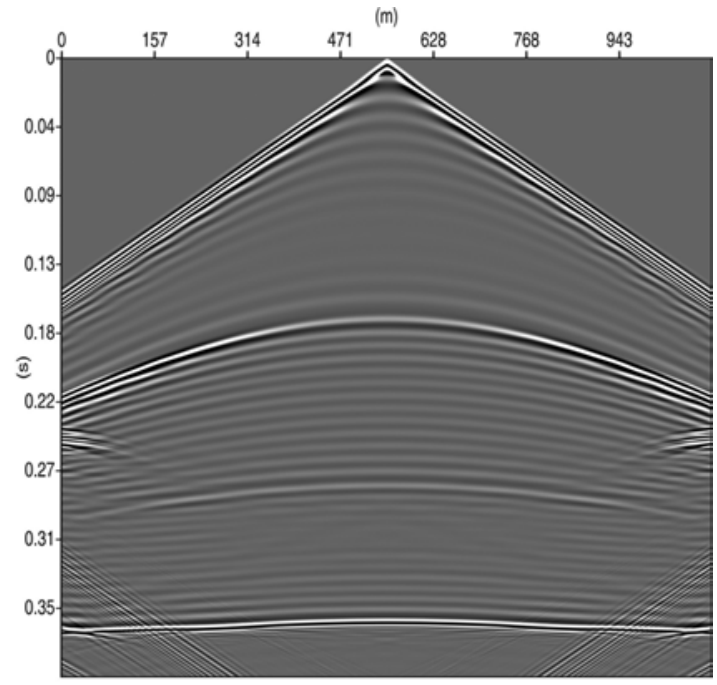

(b)

Figura 17 - Sismogramas sintéticos referentes ao modelo $M_{V I}$, usando: (a) PB, e (b) PPT. Em (a), mais uma vez, vemos que no sismograma gerado usando PPT do modelo, muitos eventos são enfraquecidos pela grande quantidade de reflexões artificiais.

des nas camadas em cada um dos modelos, pois, diferentes parametrizações aplicadas ao mesmo modelo sísmico geram modelos parametrizados diferentes. Em outras palavras, diferentes parametrizações de um mesmo modelo podem gerar novos modelos que, no nível do detalhe, podem ser distintos. Em todos os sismogramas gerados através da PPT, não foi solucionado, satisfatoriamente, o problema das reflexões nas bordas do modelo, apesar de ser utilizada a mesma técnica tanto no caso da PB como no da PPT, sendo que para a primeira obtivemos um bom resultado.

Como os modelos implementados neste trabalho possuem uma forte variação de velocidade, que dificulta 0 processo de modelagem pelo MDF, os modelos de velocidades poderiam ser suavizados, utilizando-se, por exemplo, média móvel, de modo a obtermos melhores resultados na modelagem. Entretanto, embora a parametrização possa implicar em suavização, como 
ocorre com a PPT, o contrário não ocorre. Isto é, a suavização não é um modo de representação de modelos, portanto está fora do objetivo deste trabalho, que consiste em avaliar os efeitos que diferentes representações (parametrizações), de um mesmo modelo, produzem na modelagem.

A PPT exibe ondulações na representação do modelo, isto produz falsos eventos no sismograma. Por outro lado, o uso da PB gera sismogramas com eventos bem definidos. Isto pode nos fazer pensar que a PB é superior a PPT na representação dos modelos, mas não podemos perder de vista o fato dos modelos usados neste trabalho serem esquemáticos (com extensas regiões homogêneas). Para estes modelos, como este trabalho mostra, a PB parece ser mais adequada. Entretanto, para modelos mais realísticos não podemos dizer o mesmo, pois a PB pode não ser capaz de atender ao elevado grau de complexidade do modelo e, além disso, neste caso, não existem garantias de que o sismograma exibirá eventos menos espúrios. Deve-se acrescentar ainda, que as oscilações da PPT podem ser reduzidas melhorando-se a acurácia dos cálculos para a determinação dos coeficientes ou aumentando-se o número destes, principalmente se o modelo for contínuo ou com fracas descontinuidades.

0 uso da PPT na representação de vagarosidades pode ser menos oscilante do que na representação de velocidades, desde que estas nunca sejam menores do que $1,0 \mathrm{~km} / \mathrm{s}$, pois, neste caso, as oscilações serão insuportavelmente altas. Além disso, para velocidades altas, a vagarosidade diminui drasticamente, e ela encontra-se elevada ao quadrado no denominador da fórmula recursiva (20) usada no MDF. Isto pode causar grandes instabilidades numéricas no referido método.

\section{CONCLUSÕES}

Em termos gerais, o algoritmo implementado por diferenças finitas (DF) produziu bons resultados tanto para a parametrização por blocos (PB) quanto para a parametrização polinomial trigonométrica (PPT).

Tanto na PB como na PPT, os sismogramas obtidos apresentaram similaridades gerais nos resultados. Reflexões artificiais presentes nos sismogramas gerados com a PPT foram capazes de esconder muitos eventos que se manifestaram de forma clara quando a PB foi usada. Tais reflexões artificiais se devem ao fato da PPT apresentar oscilações.

A PPT proporciona uma maior economia de espaço de memória do computador, devido ao fato de não necessitar ter, como entrada no programa de DF, um arquivo de velocidades, pois estas, podem ser calculadas durante a execução do pro- grama, bastando entrar com a função polinomial trigonométrica que é definida por um número relativamente pequeno de coeficientes.

Pelo menos todo o modelo heterogêneo e isotrópico pode ser representado, com o grau de exatidão desejado, pela PPT.

A PPT simplifica a representação de modelos mais complicados, de maneira que, independentemente de sua complexibilidade, estes modelos podem ser divididos em pequenos blocos, onde se realiza as integrações necessárias para obtenção dos coeficientes, como foi feito para os modelos $M_{V}$ e $M_{V I}$.

A PPT para modelos com extensas regiões de velocidade homogênea, tais como os estudados neste trabalho, revelou-se menos adequado do que a PB. Entretanto, acredita-se que esta situação possa ser revertida ao considerar-se modelos mais complicados. Um estudo deste gênero poderá ser considerado em desenvolvimentos futuros.

\section{AGRADECIMENTOS}

Agradecemos à Agência Nacional de Petróleo (ANP) pelo apoio financeiro em forma de bolsa de estudos.

\section{REFERÊNCIAS}

ALFORD R, KELLY K \& BOOR D. 1974. Accuracy of finite-difference modeling of the acoustic wave equation. Geophysics, 6: 834-842.

ALTERMAN Z \& KARAL FC. 1968. Propagation of elastic wave in layered media by finite difference methods. Bull. Seis. Soc., 58: 367-398.

B00R DM. 1972. Finite-Difference Methods for Seismic Wave Propagation in Heterogeneous Materials. Academic Press, New York.

CERJAN J, KOSLOFF D, KOSLOFF R \& RESHEF M. 1985. A nonreflecting boundary condition for discrete acoustic and elastic wave equations. Geophysics, 50: 705-708.

ČERVENÝ V, MOLOTOV I \& PŠENČíK I. 1977. Ray Method in Seismology. Univ. Karlova, Praha.

CUNHA PEM \& MUFTI I. 1997. Um Método Eficiente para Migração Pré-Empilhamento 3-D em Profundidade Reversa no Tempo pelo Método das Diferenças Finitas. Diss. de Mestrado, Universidade Federal da Bahia, Salvador, Brasil.

HILTERMAN FJ. 1970. Three-dimensional seismic modeling. Geophysics, 35: 1020-1037.

KELLER JB. 1962. Geometrical theory of diffraction. J. Opt. Soc. Am., 52: $116-130$.

KREIDER D, KULLER RC, OSTBERG DR \& PERKINS FW. 1972. Introdução à Análise Linear 2. Editora Universal de Brasília, Rio de Janeiro, 436-442. 
MITCHELL AR. 1969. Computational Methods in Partial Differential Equations. John Wiley and Sons, London.

OTTAVIANE M. 1971. Elastic wave propagation in two evenly welded quarter spaces. Bull. Seis. Soc. Am., 61: 1119-1152.

TROREY AW. 1970. A simple theory for seismic diffractions. Geophysics, 35: 762-784.

\section{NOTAS SOBRE OS AUTORES}

Roberto Hugo Melo dos Santos graduou-se em Matemática pela UNIT-SE (Universidade Tiradentes, Sergipe) em 1998. Obteve seu mestrado em Geofísica de Exploração de Petróleo na Universidade Federal da Bahia (UFBA) em 2002. Atualmente é aluno do curso de doutorado em Geofísica da UFBA. Suas principais áreas de interesse são: Modelagem, Inversão e Processamento de Dados Sísmicos.

Wilson Mouzer Figueiró bacharelou-se em Matemática na Universidade Federal do Rio de Janeiro (UFRJ) em 1983. Ingressou como docente no Departamento de Geologia e Geofísica Aplicada (DGGA) do Instituto de Geociências (IGEO) da Universidade Federal da Bahia (UFBA) em 1992, onde, desde então, leciona Geofísica Matemática. Doutorou-se em Geofísica na UFBA em 1994. Realizou estudos de Pós-doutorado no "Département Terre-Atmosphère-Océan de L'Ecole Normale Supérieure de Paris" (DTA0-ENS), França, no período 1997-1999. É pesquisador do Centro de Pesquisa em Geofííca e Geologia (CPGG) da UFBA. Suas áreas de interesses são: Modelagem, Inversão, Traçamento de Raios Sísmicos, e Matemática Aplicada às Geociências. 